\title{
Improvement of vertical and residual velocities in pressure or hybrid sigma-pressure coordinates in analysis data in the stratosphere
}

\author{
I. Wohltmann and M. Rex \\ Alfred Wegener Institute for Polar and Marine Research, Potsdam, Germany \\ Received: 6 September 2007 - Published in Atmos. Chem. Phys. Discuss.: 13 September 2007 \\ Revised: 28 November 2007 - Accepted: 9 December 2007 - Published: 21 January 2008
}

\begin{abstract}
Stratospheric vertical winds from analysis data in pressure $(p)$ or hybrid pressure $(\sigma-p)$ coordinates, for use in e.g. chemical transport models (CTMs) or trajectory models, often suffer both from excessive noise and errors in their mean magnitude, which in turn can introduce errors in important dynamical quantities like vertical mixing or constituent transport with the residual circulation. Since vertical velocities cannot be measured directly, they are inferred from other quantities, typically from horizontal wind divergence, that is the mass continuity equation. We propose a method to calculate the vertical wind field from the thermodynamic energy equation in $p$ or $\sigma$ - $p$ vertical coordinates that substantially reduces noise and overestimation of the residual circulation. It is completely equivalent to the approach using potential temperature $(\theta)$ as a vertical coordinate and diabatic heating rates as vertical velocities, which has already been demonstrated to give superior results to the continuity equation. It provides a quickly realizable improvement of the vertical winds, when a change of the vertical variable would cause an inadequate effort (e.g. in CTMs). The method is only applicable for stably stratified regions like the stratosphere.
\end{abstract}

\section{Introduction}

Analysis and reanalysis data from e.g. the European Centre for Medium-Range Weather Forecasts (ECMWF) (Uppala et al., 2005), the United Kingdom Meteorological Office (UKMO) (Swinbank and O'Neill, 1994) or the NASA Goddard Earth Observation System (GEOS) (Schoeberl et al., 2003) often suffer from noisy and biased vertical winds based on the continuity equation in $p$ coordinates. In turn, too much vertical dispersion and mixing or problems with the residual circulation and the mean age of air are introduced

Correspondence to: I. Wohltmann

(iwohltmann@awi-potsdam.de) (e.g. Uppala et al., 2005). While there are promising developments in new datasets as the ERA-Interim reanalysis (Monge-Sanz et al., 2007), there is a need to improve the analysis data in these aspects.

Vertical wind is an issue in CTMs, which use these data to run the dynamical part of the model. For example, a too young age of air and a too rapid residual circulation are reported for CTMs using GEOS analysis data (and even General Circulation Model (GCM) data) in Hall et al. (1999) or for TM3 in Bregman et al. (2003) (ECMWF forecast data) and van Noije et al. (2004) (ECMWF ERA-40). Excessive vertical dispersion and problems with the age of air are reported in Schoeberl et al. (2003) for the GEOS Finite Volume Data Assimilation System (FVDAS) and UKMO. Models like SLIMCAT (Chipperfield, 2006) (UKMO or ECMWF) or IMATCH (Mahowald et al., 2002) are not affected in the same way, since they use $\theta$ coordinates and calculate vertical movements by diabatic heating rates. Indeed, a better performance of this $\theta$ coordinate approach in comparison to the continuity equation in $p$ or $\sigma-p$ coordinates has been demonstrated for trajectory calculations, CTMs and even for the internally consistent wind and temperature fields of GCMs (Eluszkiewicz et al., 2000; Mahowald et al., 2002; Schoeberl et al., 2003; Chipperfield, 2006). These improvements carry over to the winds from the thermodynamic equation in $p$ or $\sigma-p$ coordinates, as we will show shortly. In addition to CTMs, there are many other studies, like trajectory calculations (e.g. Fueglistaler et al., 2005), which rely on vertical winds from analysis data and could benefit from improved vertical velocities.

The calculation of reliable vertical winds is a longstanding topic in numerical weather prediction (e.g. Krishnamurti and Bounoua, 1996). Several methods have been proposed to calculate vertical wind fields in $p$ coordinates: The "kinematic" method (vertical wind $w$ from the continuity equation), the "adiabatic" or "diabatic" method ( $w$ from the thermodynamic energy equation), the

Published by Copernicus Publications on behalf of the European Geosciences Union. 
"vorticity" method ( $w$ from the vorticity equation) and the omega equation (a combination of several equations that avoids time derivatives). We choose the diabatic method here, since it is ideally suited for the stratosphere. In addition, it is possible to derive winds for analyses with a low upper boundary (e.g. the NCEP reanalysis, Kistler et al., 2001) if the radiative transfer above this boundary is sufficiently constant, which is difficult with the continuity equation.

\section{Eulerian vertical winds}

Usually, vertical wind is obtained from the continuity equation

$\nabla \cdot\left(\rho_{0} \mathbf{u}\right)=0$

which describes the conservation of mass in $p$ coordinates. $\mathbf{u}=(u, v, w)$ is the vector of zonal wind $u$, meridional wind $v$ and vertical wind $w$ in spherical coordinates. All following equations will use log-pressure height $z=-H \log \left(p / p_{0}\right)$ as vertical coordinate ( $p$ pressure, $p_{0}$ reference pressure, $H=R T_{0} / g$ scale height, $R$ gas constant, $T_{0}$ reference temperature, $g$ gravitational acceleration), for which $\rho_{0}=p_{0} /\left(R T_{0}\right) \exp (-z / H)$ is the air density. Solving for $w$ gives

$w(z)=\frac{1}{\rho_{0}(z)} \int_{z}^{z_{\infty}} \rho_{0}\left(z^{\prime}\right) \nabla_{h} \cdot \mathbf{u}_{h}\left(z^{\prime}\right) \mathrm{d} z^{\prime}$

where $\nabla_{h} \cdot \mathbf{u}_{h}(z)=\left[\partial_{\lambda} u(z)+\partial_{\varphi}(v(z) \cos \varphi)\right] /(a \cos \varphi)$ is the horizontal wind divergence in spherical coordinates $(\lambda$ longitude, $\varphi$ latitude, $a$ earth radius, $\partial$ Eulerian derivative). $z_{\infty}$ is the log-pressure height of the highest given altitude level. The upper boundary condition is assumed to be $w\left(z_{\infty}\right)=0$ here. If $u$ and $v$ are given, $w$ can be calculated.

However, the continuity equation is not the only conservation equation one can use to determine the vertical wind. At the same time, energy needs to be conserved by the first law of thermodynamics, expressed by the near conservation of potential temperature $\theta=T\left(p_{0} / p\right)^{2 / 7}$ (with $T$ temperature), which can only be changed by radiative heating $Q$

$$
\frac{\mathrm{D} \theta}{\mathrm{D} t}=Q
$$

$\mathrm{D} / \mathrm{D} t$ is the Lagrangian derivative. Solving for $w$ gives

$$
w=\left(Q-\partial_{t} \theta-\frac{u}{a \cos \varphi} \partial_{\lambda} \theta-\frac{v}{a} \partial_{\varphi} \theta\right) / \partial_{z} \theta
$$

If $Q, T, u$ and $v$ are given, $w$ can be calculated (note that we do not use Eq. (4) in this study, but the method presented in the next section). $Q$ is obtained by a radiative transfer model, which needs $T$ profiles as input data. Since we divide by the static stability $\partial_{z} \theta$, the equation can only be used in stably stratified regions like the stratosphere. For short time-scales, the diabatic component $Q / \partial_{z} \theta$ of the wind can usually be neglected, while it is the most important term on time-scales of several months.

In theory, Eqs. (2) and (4) obviously should give identical results. However, since $u, v$ and $T$ are measured quantities prone to errors and data are discretized and interpolated, Eqs. (2) and (4) will not be fulfilled at the same time in practice. This also means that mass is not conserved if Eq. (4) or the approach in the next section is used to calculate vertical winds. To ensure conservation of mass, a procedure similar to that presented in Weaver et al. (2000) can be used to correct the horizontal wind by adjusting the divergence to be zero while conserving the vorticity of the wind field (see Appendix B for explicit equations). We do not follow this approach here, since the implied changes to the horizontal wind are rather small.

\section{Semi-lagrangian approach}

Equation (4) substantially reduces noise in the vertical wind fields, but is not sufficient for long-time integrations and an accurate determination of the residual circulation. Since Eq. (4) basically represents an advection problem in an Eulerian framework, a criterion identical to the CourantFriedrichs-Lewy criterion $u \Delta t \leq \Delta x$ applies as a necessary condition for a stable solution, where $u$ is the advection velocity, $\Delta x$ is the grid spacing and $\Delta t$ is the time step of the analysis. Since the time step at which the data is obtained is typically $6 \mathrm{~h}$ and the grid spacing typically $2.5^{\circ}$, this condition is usually not fulfilled for available analysis data, especially at high latitudes (the time step and grid spacing of the underlying model of the analysis are usually much higher, but cannot be used due to computational constraints). Note also that since there is no exact constraint to a certain $\theta$ level as in an application with $\theta$ levels as vertical coordinate, the trajectories/air masses tend to drift away from the correct $\theta$ level even if only small systematic errors are present in the vertical wind. Error sources are e.g. the approximation of derivatives by finite differences or interpolation errors. Hence, we use a Semi-Lagrangian approach, where we calculate forward and backward trajectories in a $\theta$ coordinate system starting/ending at the analysis grid points in the $p$ or $\sigma-p$ coordinate system and use the pressure difference between the start and end points of the trajectories (divided by the travel time of the trajectories) as a direct measure for the vertical wind.

In the examples given here, vertical winds are calculated for every grid-point in longitude, latitude and time, but for a staggered grid in the $p$ or $\sigma-p$ coordinate, with the new grid points centered in log-pressure between the old levels, which greatly improves the long-term stability of the trajectories in the vertical direction (see Appendix B). At every fourdimensional grid-point, a $12 \mathrm{~h}$ forward and a $12 \mathrm{~h}$ backward 

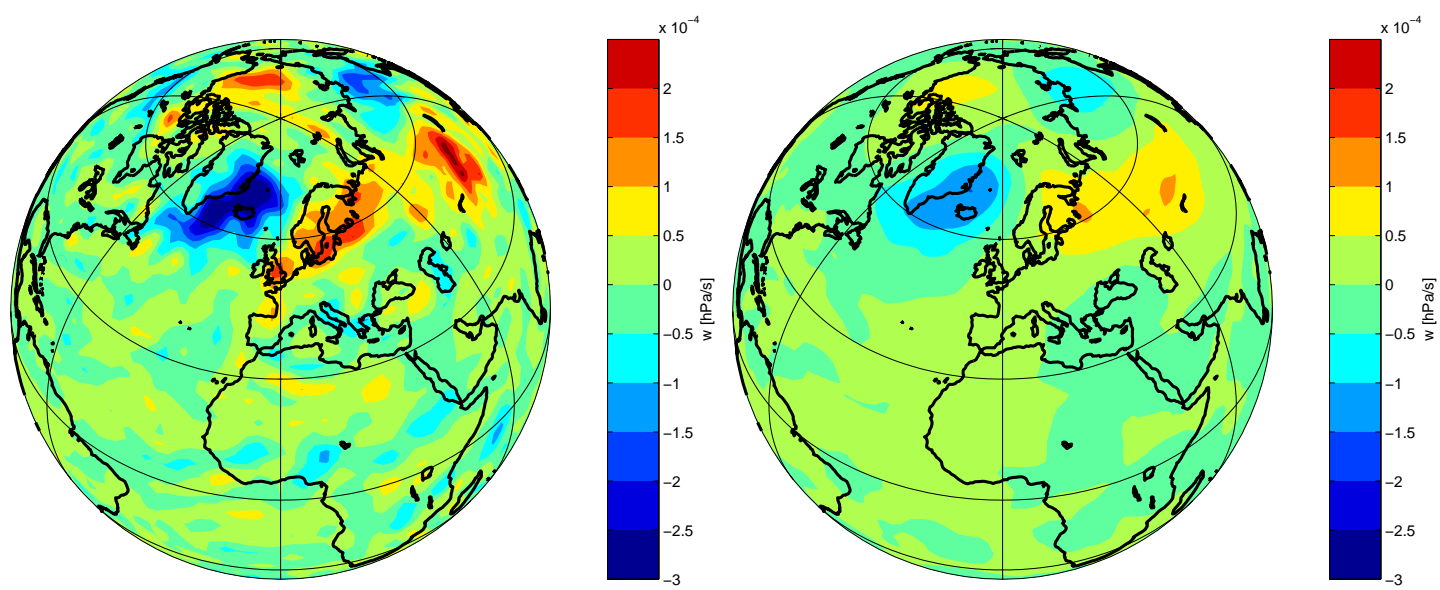

Fig. 1. Vertical wind from ERA-40 reanalysis data on standard pressure levels, calculated with two different methods. Left panel: Vertical wind field at $50 \mathrm{hPa}$ from the continuity equation as provided by ECMWF, averaged over 1 January 2000 00:00-24:00 h (UTC) (to compare better with the right panel). Right panel: Vertical wind field from the thermodynamic equation and the Semi-Lagrangian approach for the staggered level between 30 and $50 \mathrm{hPa}$ and 1 January 12:00 $\mathrm{h}$ (UTC).

trajectory in $\theta$ coordinates are started. The pressure difference between the latest date of the forward trajectory and the earliest date of the backward trajectory divided by $24 \mathrm{~h}$ is the vertical wind at the grid-point. Potential temperature on the isentropic forward and backward trajectories is only allowed to change by radiative heating. The $24 \mathrm{~h}$ period was determined empirically as a compromise between the temporal resolution of the winds and the stability of the method, which gets worse for shorter time periods.

The isentropic trajectory model uses a 4th order RungeKutta method for integration with a 10 min time step. Spherical coordinates are used, but poleward of $85^{\circ}$, the projection is switched to a polar projection to avoid the singularities at the poles. Wind and temperature values are interpolated linearly in longitude, latitude, the logarithm of $\theta$ and time to the position of the trajectory at every time step. Pressure is determined from the interpolated temperature and $\theta$ by solving $\theta=T\left(p_{0} / p\right)^{2 / 7}$ for $p$. Tests with cubic interpolation showed no significant differences in the results.

Note that the original meteorological data set, which is given either at $\sigma-p$ or $p$ levels, is not transformed to an intermediate data set with several fixed $\theta$ levels as vertical coordinate here, which would introduce additional and unnecessary interpolations. Instead, the trajectory model calculates $\theta$ at each original grid point of the meteorological data set and interpolates locally to the isentrope where the trajectory currently is located (see Appendix C).

\section{Results and discussion}

The left panel of Fig. 1 shows a vertical wind field derived from the continuity equation, as provided by the ECMWF ERA-40 reanalysis $(50 \mathrm{hPa}$ taken from the standard pressure levels, horizontal $2.5^{\circ} \times 2.5^{\circ}$ grid, 1 January 2000 averaged over 00:00-24:00 h UTC to better compare with the right panel). The right panel shows a field calculated from the thermodynamic equation with the Semi-Lagrangian method that closely simulates the wind field in the left panel (calculated with ERA-40 temperature and wind data at the same day at 12:00 h UTC with $12 \mathrm{~h}$ back- and forward trajectories, staggered level between 30 and $50 \mathrm{hPa}$ ). It is obvious that the right panel is considerably less noisy.

It could be argued that the scatter is real and that the left panel is the correct one. However, the vertical winds in the left panel would correspond to heating rates of several $\mathrm{K} /$ day after subtraction of the part of the wind that is caused by adiabatic movements. Such heating rates can be ruled out as unphysical (see also Eluszkiewicz et al., 2000). In addition, the vertical mixing caused by these winds would be much larger than observed (see discussion of diffusion coefficients below).

The method is now tested with ERA-40 $\sigma-p$ model level data (60 levels) with a horizontal resolution of $2^{\circ} \times 2^{\circ}$. After calculating the Eulerian vertical winds with the SemiLagrangian method using these ERA-40 data, the original vertical winds from ERA-40 are replaced on a staggered grid, while keeping the horizontal winds and temperature. This data set is used to drive several test runs with a trajectory model using the pressure of the model levels as coordinate (THERMO-P hereafter, red dots in the following figures). In addition, results are compared with trajectory runs with the original vertical winds from the continuity equation in the same coordinate system (CONT-P, blue dots) and with the potential temperatures of the model levels as the coordinate and heating rates as vertical velocities (Q-THETA, green dots). 
Heating rates are obtained directly from the ERA-40 archive and are based on the radiative transfer model in use by ECMWF (Morcrette et al., 1998), which uses climatological ozone and prognostic water vapor profiles in the stratosphere. Note that there are known temperature biases and fluctuations compared to measurements in ERA-40 data (Uppala et al., 2005), which could affect the heating rates.

The isobaric trajectory model using vertical winds (used for the THERMO-P and CONT-P cases) is largely identical to the isentropic trajectory model using heating rates (used for the calculation of the Semi-Lagrangian thermodynamic winds and the Q-THETA case). The only difference is that the vertical interpolation is in the logarithm of $p$ and not in the logarithm of $\theta$.

As a first example we start forward trajectory runs at the equator. For all runs, 1440 trajectories are started at $0^{\circ} \mathrm{N}$ on 1 January 2000 (00:00 h UTC), equally spaced in longitude in $0.25^{\circ}$ steps at the $475 \mathrm{~K}$ isentropic level. Trajectories are integrated for 20 days.

To test the long-term stability of the method first, we run this setup with a prescribed artificial constant heating rate of $0 \mathrm{~K} /$ day. Results after 20 days show an average of $475.5 \mathrm{~K}$ and a standard deviation of $3.4 \mathrm{~K}$ over all trajectory endpoints. A run with $1 \mathrm{~K} /$ day heating shows an average of $495.7 \mathrm{~K}$ and a standard deviation of $3.2 \mathrm{~K}$. Integration periods of up to 100 days show similar results. This demonstrates the stability of the method.

Figure 2 (left) shows results for the three combinations of vertical coordinates and velocities described above. The Lagrangian mean over the difference of the vertical start and end positions of the trajectories is a direct measure for the vertical residual velocity of the tropical upward branch of the Brewer-Dobson circulation (Andrews et al., 1987). Results are shown in Table 1. The difference between the continuity equation and the thermodynamic equation is noticeable. The mean upward velocity is $15.5 \mathrm{~K}$ in 20 days for CONT-P and $10.2 \mathrm{~K}$ in 20 days for THERMO-P. Q-THETA shows a change of $8.9 \mathrm{~K}$, comparable to THERMO-P. The table also contains calculations based on standard pressure level data instead of model level data (everything else being the same). The difference between the continuity and thermodynamic equation is much less pronounced here, which demonstrates that many other parameters, like the number of levels, can significantly influence the results.

Additionally, the table shows the results when averaging the CONT-P vertical winds over $24 \mathrm{~h}$ (running average over 5 analysis time steps) to concur with the $24 \mathrm{~h}$ trajectories of the THERMO-P case. Results for the CONT-P vertical winds averaged over $24 \mathrm{~h}$ and horizontally over the nearest 9 grid points (including the original point) are also shown in the table, since it could be argued that there is additional spatial smoothing in the THERMO-P winds from the interpolation to the trajectory points. This has only a moderate effect on the mean upward velocity, which is now $13.3 \mathrm{~K}$ or
$17.1 \mathrm{~K}$. The mean upward velocity is consistently higher in the CONT-P runs than in the other runs.

Results for the vertical residual velocity from the trajectory calculations are compared to observed vertical velocities in geopotential height (Mote et al., 1998) and potential temperature (Hall and Waugh, 1997), inferred from the tape recorder signal in tropical stratospheric water vapor mixing ratios. Typical heating rates in the tropical stratosphere are in the order of $10 \mathrm{~K}$ in 20 days (e.g. Hall and Waugh, 1997), which compares well with the THERMO-P and Q-THETA cases, while the three $\sigma$ - $p$ CONT-P cases are about 50\% higher on average. Comparison with Mote et al. (1998) is only possible for standard pressure levels, since the model level data contains no geopotential. CONT-P shows a mean change of $378 \mathrm{gpm}$ in geopotential height, while THERMO$P$ shows a change of $397 \mathrm{gpm}$ and Q-THETA shows a change of $342 \mathrm{gpm}$. Mote et al. (1998) give long-term mean vertical speeds of $0.2 \mathrm{~mm} / \mathrm{s}$ at altitudes of about $20 \mathrm{~km}$ for HALOE data, corresponding to $345 \mathrm{gpm}$ in 20 days. Niwano et al. (2003) also suggests a value near $0.2 \mathrm{~mm} / \mathrm{s}$. All values agree roughly within the uncertainties of the observations and our calculations.

The runs can also be used to derive the vertical eddy diffusion coefficient $K_{z}$, since $K_{z}$ and the standard deviation $\sigma$ of the end-points of the trajectories are related by $K_{z}=\sigma^{2} /(2 t)$, where $t$ is the integration time (this follows from Fick's law with a delta function as initial condition). Table 1 shows observed values derived from the tape recorder signal in comparison to the values inferred from the trajectory runs. The vertical diffusion coefficients derived from the continuity equation are more than two orders of magnitude larger than observed and are clearly outside the possible range of $K_{z}$ values compatible with the observed tape recorder signal (Hall and Waugh, 1997; Mote et al., 1998). The diffusion coefficients from the isentropic and thermodynamic runs are much closer to reality. They somewhat underestimate the observed values, perhaps due to missing sub-grid processes.

The result for the both CONT-P cases with averaged winds is surprising: The standard deviation of the end-points of the trajectories is only slightly reduced compared to the run with the instantaneous winds, and gives a $K_{Z}$ only $20 \%-40 \%$ smaller than for the CONT-P run with instantaneous winds. In contrast, the standard deviation of the vertical winds itself (on a given level and date as in Fig. 1) is reduced by about a factor of 2 in the $24 \mathrm{~h}$ averaged case, as expected. This points to spurious fluctuations with longer time scales than $24 \mathrm{~h}$ in the vertical wind field or other systematic problems. However, it is not related to the much larger amplitude of the vertical winds in the CONT-P case compared to the QTHETA case, which could lead to more interpolation error. The larger amplitude is caused by the adiabatic component of the wind, which is large compared to the diabatic component. However, this wind component is also present in the THERMO-P case, which shows a much smaller $K_{z}$.

Note that it is very difficult to decide what would be a fair 
Table 1. Performance of different representations of the vertical wind field. Vertical velocities are derived from the continuity equation (CONT-P) in pressure coordinates, the thermodynamic equation (THERMO-P) in pressure coordinates or from heating rates (Q-THETA) in $\theta$ coordinates (all coordinates are interpolated both from standard pressure levels $p$ or model levels $\sigma$ - $p$ ). For CONT-P on model levels, results are given for three cases: instantaneous vertical winds directly from the analysis data, winds averaged over $24 \mathrm{~h}$ (running average over 5 analysis time steps) and winds averaged over $24 \mathrm{~h}$ and additionally spatially over the nearest 9 grid points. 2nd and 3rd column: Mean ascent (1-21 January 2000) in the tropics based on geopotential height or potential temperature (from Fig. 2, left). 4th and 5th column: Eddy diffusion coefficients $K_{z}$ based on geopotential height or potential temperature (from Fig. 2, left). 6th column: Mean descent (26 November 1999 to 5 March 2000) in the polar vortex in potential temperature (from Fig. 2, right). All values are compared to observations: ${ }^{\mathrm{a}}$ Hall and Waugh (1997), ${ }^{\mathrm{b}}$ Mote et al. (1998), ${ }^{\mathrm{c}}$ Greenblatt et al. (2002).

\begin{tabular}{lccccc}
\hline & \multicolumn{3}{c}{ Ascent tropics } & \multicolumn{2}{c}{$K_{z}$ tropics } \\
& $(\mathrm{K})$ & $(\mathrm{m})$ & $\left(\mathrm{K}^{2} / \mathrm{d}\right)$ & $\begin{array}{c}\text { Descent } \\
\left(\mathrm{m}^{2} / \mathrm{s}\right)\end{array}$ & vortex $(\mathrm{K})$ \\
\hline Observed & $10^{\mathrm{a}}$ & $345^{\mathrm{b}}$ & $0.3^{\mathrm{a}}$ & $0.02^{\mathrm{b}}$ & $63^{\mathrm{c}}$ \\
CONT-P $p$ (instantaneous) & 9.8 & 378.2 & 37.3 & 0.47 & 205.7 \\
CONT-P $\sigma-p$ (instantaneous) & 15.5 & - & 54.7 & - & 186.6 \\
CONT-P $\sigma-p(24 \mathrm{~h})$ & 13.3 & - & 42.2 & - & 180.9 \\
CONT-P $\sigma-p(24 \mathrm{~h}+$ spatial) & 17.1 & - & 32.2 & - & 258.1 \\
THERMO-P $p$ & 11.7 & 396.9 & 0.24 & 0.001 & 91.4 \\
THERMO-P $\sigma-p$ & 10.2 & - & 0.25 & - & 44.0 \\
Q-THETA $p$ & 10.0 & 342.4 & 0.009 & 0.002 & 73.1 \\
Q-THETA $\sigma-p$ & 8.9 & - & 0.04 & - & 47.5 \\
\hline
\end{tabular}

comparison between the CONT-P winds and the THERMO$\mathrm{P}$ winds, since the method of calculation is fundamentally different and involves different interpolations and averages at different locations and dates. For example, there is an average over several pressure levels and several derivatives in the divergence operator in the continuity equation, which should also be considered. However, the question how well our method performs in comparison to approaches actually used in existing models is more important than a completely fair comparison in the end.

Figure 2 (right) shows results of backward trajectory runs in the polar vortex as a second example. For all runs, trajectories are initialized on a $2.5^{\circ} \times 2.5^{\circ}$ grid inside the polar vortex at the $450 \mathrm{~K}$ isentropic level. The polar vortex is defined as the area inside the 20 PVU contour of Lait's modified potential vorticity $\left(\theta_{0}=420 \mathrm{~K}\right)$ (Lait, 1994). Trajectories start on 5 March 2000 (12:00 h UTC) and run for 100 days until 26 November 1999. The winter 1999/2000 is selected because it is one of the few winters in which tracer measurements are available for comparison.

The plot shows the position of the trajectories on 26 November 1999 (12:00 $\mathrm{h}$ UTC) as a function of modified $\mathrm{PV}$ and $\theta$. Only trajectories inside the 20 PVU contour on 1 January 2000 and inside the 15 PVU contour on 26 November 1999 are shown (basically trajectories that stayed inside the vortex). The trajectories show a much larger vertical dispersion in the case of the continuity equation again.

The Lagrangian mean over the difference of the vertical start and end positions of all trajectories is now a measure for the vertical residual velocity of the polar downward branch of the Brewer-Dobson circulation. The mean downward ve- locity from 26 November 1999 to 5 March 2000 shows relatively large differences between the three $\sigma-p$ CONT-P runs $(\Delta \theta=187,181,258 \mathrm{~K})$, which may be related to the spurious oscillations in ERA-40 data in the polar stratosphere (Uppala et al., 2005). However, the most obvious difference is between the CONT-P runs and the THERMO-P run $(\Delta \theta=44 \mathrm{~K})$. Results for the averaged vertical velocity are compared to descent rates inferred from tracer measurements of $\mathrm{N}_{2} \mathrm{O}$ (Greenblatt et al., 2002). $\mathrm{N}_{2} \mathrm{O}$ tracer measurements conducted around 26 November 1999 (solid black line, $\theta=513 \mathrm{~K}$ ) and around 5 March 2000 (dashed black line, $\theta=450 \mathrm{~K}$ ) give a change of $\Delta \theta=63 \mathrm{~K}$. In comparison to this value, the value from the THERMO-P run is far more realistic than the values from the CONT-P runs, which overestimate the descent rates by a factor of 3. The Q-THETA run $(\Delta \theta=48 \mathrm{~K})$ compares well with the THERMO-P run, but both runs show values slightly too small compared to the observations. Again, there are noticeable differences if standard pressure level data is used in all runs (Table 1).

This article was inspired by the question in how far the use of vertical wind fields from continuity affected water vapor transport into the stratosphere in Fueglistaler et al. (2005). Figure 3 shows results of backward trajectory runs started on 29 February 2000 at $400 \mathrm{~K}$ on a $2^{\circ} \times 2^{\circ}$ grid between $30^{\circ} \mathrm{N} / \mathrm{S}$ and run until they reached the $365 \mathrm{~K}$ level for all three wind fields discussed above. The upper panel shows position and temperature of the coldest point along each trajectory while the lower panel shows the distribution of residence times of the trajectories between $365-375 \mathrm{~K}$. While the cold point locations remain relatively unaffected (small change in the stratospheric water vapor obtained by freeze drying), mean 

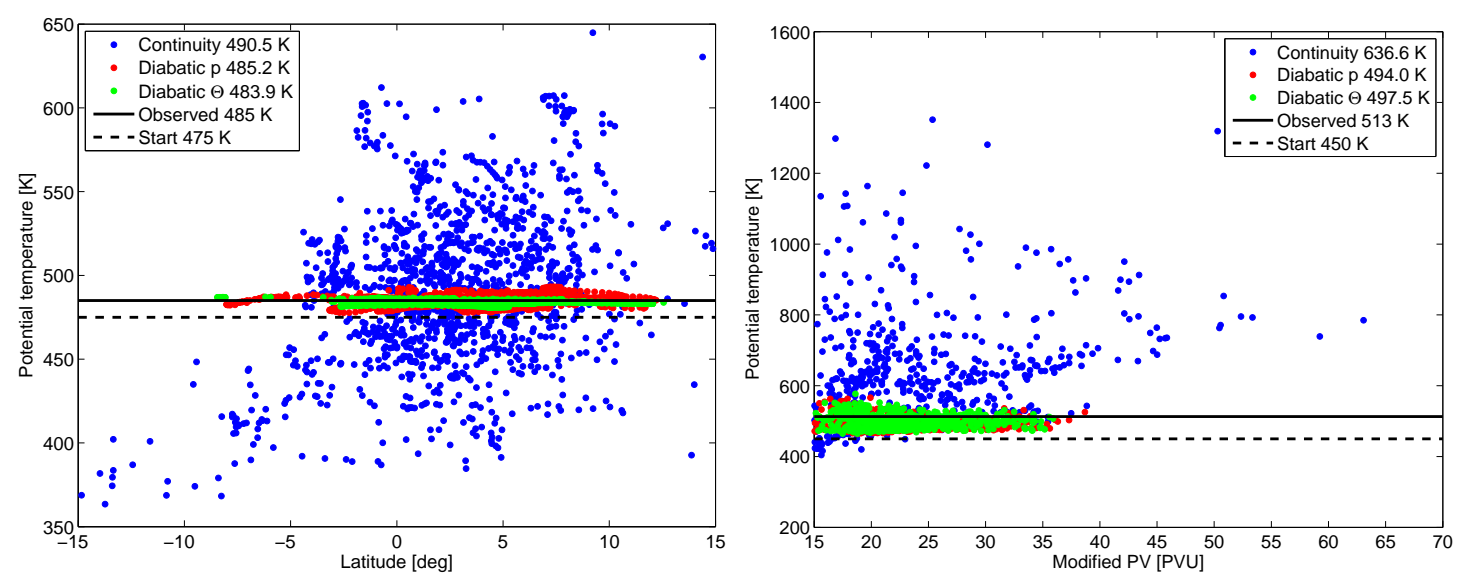

Fig. 2. Trajectory runs driven with different vertical wind fields. Left: Position of 1440 forward trajectories started at $1 \mathrm{January} 2000$ (00:00 h UTC) on the equator after 20 days, for winds from the continuity equation in pressure coordinates (blue dots), from the thermodynamic equation in pressure coordinates (red dots) and from heating rates in $\theta$ coordinates (green dots). Right: Position of backward trajectories on 26 November 1999 (12:00 h UTC) started at $450 \mathrm{~K}$ inside the polar vortex on a 2.5 ${ }^{\circ}$ grid on $5 \mathrm{March}$ 2000 (12:00 h UTC).

residence times differ by a factor of 2 , which directly affects chemical and microphysical processing.

\section{Conclusions}

We propose a new method to calculate vertical wind fields from analysis data based on the thermodynamic equation. It substantially reduces overestimation of the residual circulation and spurious noise that usually leads to an overestimation of vertical diffusion by several orders of magnitude (compared to wind fields based on the continuity equation as usually given in analysis data). In contrast, temporal or spatial averaging of the winds from the continuity equation does not significantly improve their performance.

The method proposed here is thought mainly for the application in chemical transport modelling or trajectory models, which use off-line meteorological data and could increase the quality of such models. The method is easily applied in existing models by just exchanging the vertical wind field that is used as input data without changing any model code. On request, we will provide vertical wind fields for modelling studies (see email address). The examples show the importance of a correct representation of vertical wind fields in modelling studies, which will remain an issue in the future.

\section{Appendix A}

\section{Correction of horizontal winds}

It may be desirable to conserve mass and energy at the same time. This is possible if we correct the horizontal wind for mass conservation after calculating the vertical wind from the thermodynamic equation. These corrections are small compared to the magnitude of the horizontal wind, so that we do not run into inconsistencies by changing the horizontal wind field too much. The following method is similar to that proposed in Weaver et al. (2000), which is not given there in mathematical detail.

Let us call the new winds $\mathbf{u}^{N}=\left(u^{N}, v^{N}, w^{N}\right)$ (with $w^{N}$ as the vertical wind from the thermodynamic equation and $u^{N}$, $v^{N}$ as the corrected horizontal winds we are looking for) and the old winds $\mathbf{u}=(u, v, w)$ (with $w$ as the vertical wind from the continuity equation and $u$ and $v$ as the horizontal winds from the analysis). For both wind vectors, mass should be conserved

$$
\nabla \cdot\left(\rho_{0} \mathbf{u}\right)=0 \quad \nabla \cdot\left(\rho_{0} \mathbf{u}^{N}\right)=0
$$

This condition is not sufficient to determine the new wind field. In addition, we demand that the curl of the wind field is not changed

$\nabla \times \mathbf{u}=\nabla \times \mathbf{u}^{N}$

In our case, $u, v, w$ and $w^{N}$ are given, while $u^{N}$ and $v^{N}$ are unknown. If $u^{\prime}=u^{N}-u, v^{\prime}=v^{N}-v$ and $w^{\prime}=w^{N}-w$ are the differences between the wind fields, $u^{\prime}$ and $v^{\prime}$ are unknown and $w^{\prime}$ is given. We need two equations to solve for the two variables $u^{\prime}$ and $v^{\prime}$. The first one is deduced from Eq. (A1) and states that for the two-dimensional divergence

$\nabla_{h} \cdot\left(u^{\prime}, v^{\prime}\right)=D$

where

$D=-\partial_{z}\left(\rho_{0} w^{\prime}\right) / \rho_{0}=-\nabla \cdot\left(u, v, w^{N}\right)$ 

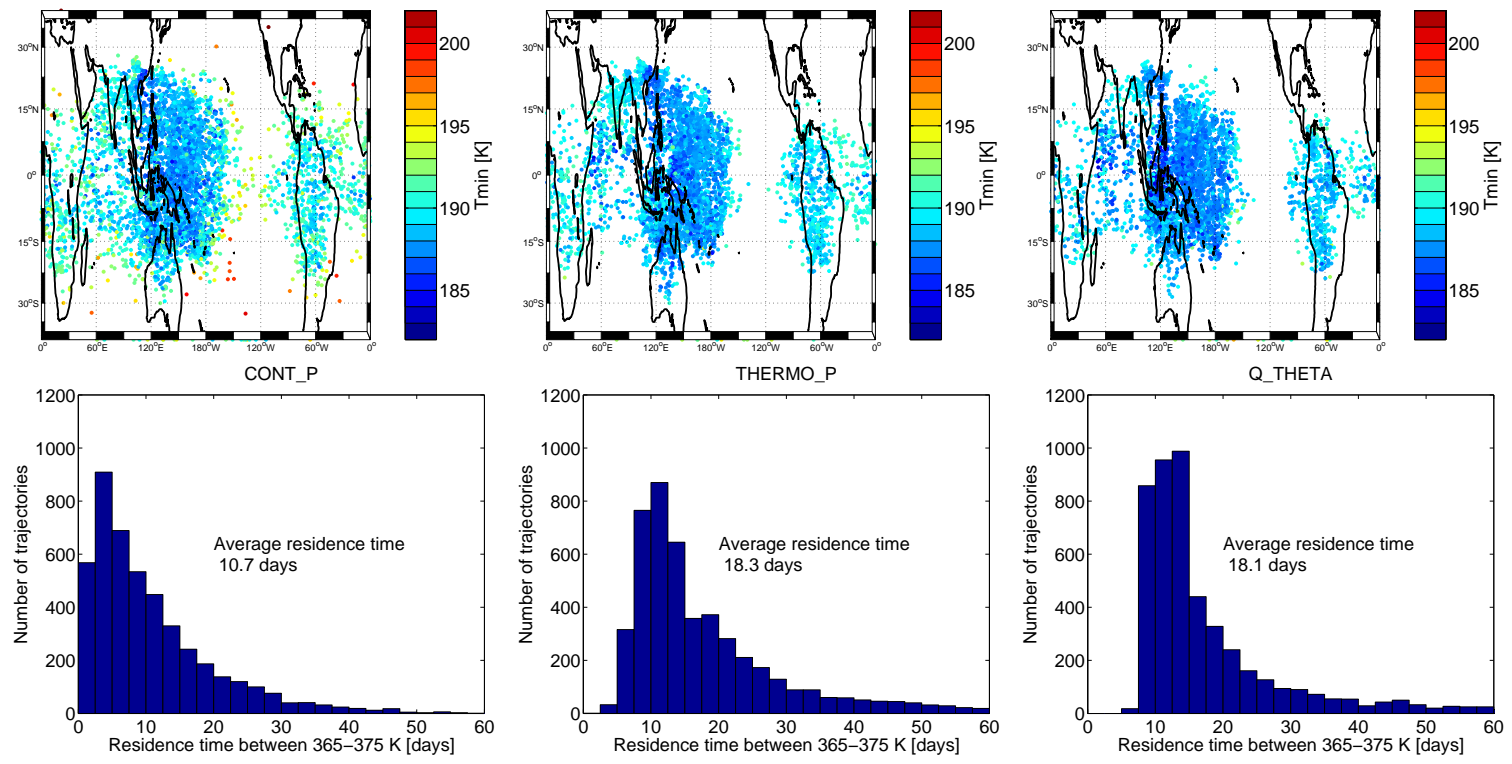

Fig. 3. Upper panel: Cold point locations and temperatures for the CONT-P, THERMO-P and Q-THETA winds and backward trajectories started on 29 February 2000 at $400 \mathrm{~K}$ on a $2^{\circ} \times 2^{\circ}$ grid between $30^{\circ} \mathrm{N} / \mathrm{S}$. Lower panel: Distribution of residence times between $365-375 \mathrm{~K}$ for the same winds.

is a known function. The second one is deduced from Eq. (A2) and states that the two-dimensional curl ( $z$ element of the three-dimensional curl) is zero

$\nabla \times\left(u^{\prime}, v^{\prime}\right)=0$

It follows that $\left(u^{\prime}, v^{\prime}\right)$ can be written as the gradient of a scalar field $\psi$

$\nabla \psi=\left(u^{\prime}, v^{\prime}\right)$

which gives a differential equation for $\psi$

$\Delta \psi=D$

This is a Poisson equation on the surface of a sphere, which has to be solved by one of the standard methods for boundary value problems. After solving for $\psi, u^{\prime}$ and $v^{\prime}$ can be determined by derivation

$u^{\prime}=\frac{\partial_{\lambda} \psi}{a \cos \varphi} \quad v^{\prime}=\frac{\partial_{\varphi} \psi}{a}$

\section{Appendix B}

\section{The staggered grid}

The staggered grid greatly improves the stability of the method and reduces systematic errors in the wind field that cause air masses to drift from the correct isentrope. The reason for this is that the number of vertical levels involved in the calculation is reduced. For example, the vertical wind at the staggered grid-point between the original levels $i$ and $i+1$ will very likely be calculated only with the pressure, wind and temperature values at the levels $i$ and $i+1$, because the isentropic trajectory used to calculate the wind will stay between these levels. If the wind would be calculated directly at the level $i$, values from levels $i-1, i$ and $i+1$ would be involved in the calculation. If now a trajectory is started using the calculated thermodynamic winds, it will use wind fields involving less levels. For example, if the trajectory oscillates around original level $i$, it will use winds from the staggered level between $i$ and $i+1$ and between $i-1$ and $i$, which are calculated only with values from levels $i-1, i$ and $i+1$. If the thermodynamic vertical winds were on the original levels, the trajectory would use winds from $i-1, i$ and $i+1$, which would be calculated with values from levels $i-2$ to $i+2$. Since the vertical grid is often quite coarse, this can encompass a vertical range of considerable depth.

\section{Appendix C}

\section{Interpolation in the trajectory model}

In the isentropic trajectory model, the original meteorological data set (given on $\sigma-p$ or $p$ levels) is not transformed to an intermediate data set with several fixed $\theta$ levels as vertical coordinate, which would introduce additional and unnecessary interpolations. Instead, data on the isentrope is directly interpolated from the original grid-points, which preserves the original resolution of the data set. 
Let the grid be $\left(\lambda_{i}, \varphi_{j}, \eta_{k}, t_{l}\right)$ with $\eta$ as the values of the vertical $\sigma-p$ coordinate and $i, j, k, l$ as indices for the grid dimensions. Let $(\lambda, \varphi, \theta, t)$ be the current position of the trajectory. Now, indices for the interpolation in longitude, latitude and time are determined as usual, such that $\lambda_{i} \leq \lambda<\lambda_{i+1}$, $\varphi_{j} \leq \varphi<\varphi_{j+1}$ and $t_{l} \leq t<t_{l+1}$. For the eight combinations $\left(\lambda_{i}, \varphi_{j}, t_{l}\right),\left(\lambda_{i+1}, \varphi_{j}, t_{l}\right), \ldots,\left(\lambda_{i+1}, \varphi_{j+1}, t_{l+1}\right)$ vertical indices $k_{1}, \ldots, k_{8}$ for interpolating in $\eta$ are determined separately by looking where the isentrope crosses the model levels, e.g. for $\left(\lambda_{i}, \varphi_{j}, t_{l}\right)$ the index is determined by $\theta\left(\lambda_{i}, \varphi_{j}, \eta_{k_{1}}, t_{l}\right) \leq \theta<\theta\left(\lambda_{i}, \varphi_{j}, \eta_{k_{1}+1}, t_{l}\right)$. That is, the vertical coordinate is not only dependent on a vertical index and has fixed values, but it also depends on the horizontal position and time.

Now, linear interpolation is used to obtain interpolated values, starting with the interpolation in the vertical coordinate. For example, for the interpolation of temperature at $\left(\lambda_{i}, \varphi_{j}, t_{l}\right)$ to $\theta$ :

$$
\begin{aligned}
& T\left(\lambda_{i}, \varphi_{j}, \theta, t_{l}\right)=\log \theta\left(\lambda_{i}, \phi_{j}, \eta_{k_{1}}, t_{l}\right) \\
& \quad+\frac{T\left(\lambda_{i}, \varphi_{j}, \eta_{k_{1}+1}, t_{l}\right)-T\left(\lambda_{i}, \varphi_{j}, \eta_{k_{1}}, t_{l}\right)}{\log \theta\left(\lambda_{i}, \varphi_{j}, \eta_{k_{1}+1}, t_{l}\right)-\log \theta\left(\lambda_{i}, \varphi_{j}, \eta_{k_{1}}, t_{l}\right)} \\
& \quad \cdot\left(\log \theta-\log \theta\left(\lambda_{i}, \varphi_{j}, \eta_{k_{1}}, t_{l}\right)\right)
\end{aligned}
$$

The same method is applied in the isobaric trajectory model by just replacing $\theta$ by $p$.

Acknowledgements. We thank P. Haynes and K. Krüger for helpful suggestions. We thank ECMWF for providing reanalysis data. This work is supported by the European Community through the SCOUT-O3 project.

Edited by: K. Hamilton

\section{References}

Andrews, D. G., Holton, J. R., and Leovy, C. B.: Middle Atmosphere Dynamics, Academic Press, 1987.

Bregman, B., Segers, A., Krol, M., Meijer, E., and van Velthoven, P.: On the use of mass-conserving wind fields in chemistrytransport models, Atmos. Chem. Phys., 3, 447-457, 2003, http://www.atmos-chem-phys.net/3/447/2003/.

Chipperfield, M. P.: New version of the TOMCAT/SLIMCAT offline chemical transport model: Intercomparison of stratospheric tracer experiments, Quart. J. Roy. Meteorol. Soc., 132, 11791203, doi:10.1256/qj.05.51, 2006.

Eluszkiewicz, J., Hemler, R. S., Mahlman, J. D., Bruhwiler, L., and Takacs, L. L.: Sensitivity of age-of-air calculations to the choice of advection scheme, J. Atmos. Sci., 57, 3185-3201, 2000.

Fueglistaler, S., Bonazzola, M., Haynes, P. H., and Peter, T.: Stratospheric water vapor predicted from the Lagrangian temperature history of air entering the stratosphere in the tropics, J. Geophys. Res., 110, D08107, doi:10.1029/2004JD005516, 2005.

Greenblatt, J. B., Jost, H.-J., Loewenstein, M., Podolske, J. R., Hurst, D. F., Elkins, J. W., Schauffler, S. M., Atlas, E. L., Herman, R. L., Webster, C. R., Bui, T. P., Moore, F. L., Ray,
E. A., Oltmans, S., Vömel, H., Blavier, J.-F., Sen, B., Stachnik, R. A., Toon, G. C., Engel, A., Müller, M., Schmidt, U., Bremer, H., Pierce, R. B., Sinnhuber, B.-M., Chipperfield, M., and Lefèvre, F.: Tracer-based determination of vortex descent in the 1999/2000 Arctic winter, J. Geophys. Res., 107, 8279, doi:10.1029/2001JD000937, 2002.

Hall, T., Waugh, D., Boering, K., and Plumb, R.: Evaluation of transport in stratospheric models, J. Geophys. Res., 104, 18 815$18839,1999$.

Hall, T. M. and Waugh, D.: Tracer transport in the tropical stratosphere due to vertical diffusion and horizontal mixing, Geophys. Res. Lett., 24, 1383-1386, 1997.

Kistler, R. E., Kalnay, E., Collins, W., Saha, S., White, G., Woollen, J., Chelliah, M., Ebisuzaki, W., Kanamitsu, M., Kousky, V., van den Dool, H., Jenne, R., and Fiorino, M.: The NCEP-NCAR 50year reanalysis: Monthly means CD-ROM and documentation, Bull. Amer. Meteorol. Soc., 82, 247-268, 2001.

Krishnamurti, T. N. and Bounoua, L.: An Introduction to Numerical Weather Prediction Techniques, CRC Press, 1996.

Lait, L. R.: An alternative form for potential vorticity, J. Atmos. Sci., 51, 1754-1759, 1994.

Mahowald, N. M., Plumb, R. A., Rasch, P. J., del Corral, J., Sassi, F., and Heres, W.: Stratospheric transport in a threedimensional isentropic coordinate model, J. Geophys. Res., 107, 4254, doi:10.1029/2001JD001313, 2002.

Monge-Sanz, B. M., Chipperfield, M. P., Simmons, A. J., and Uppala, S. M.: Mean age of air and transport in a CTM: Comparison of different ECMWF analyses, Geophys. Res. Lett., 34, L04801, doi:10.1029/2006GL028515, 2007.

Morcrette, J.-J., Clough, S. A., Mlawer, E. J., and Iacono, M. J.: Impact of a validated radiative transfer scheme, RRTM, on the ECMWF model climate and 10-day forecasts, ECMWF Tech. Memo., 252, 1-47, 1998.

Mote, P. W., Dunkerton, T. J., McIntyre, M. E., Ray, E. A., Haynes, P. H., and Russell III, J. M.: Vertical velocity, vertical diffusion, and dilution by midlatitude air in the tropical lower stratosphere, J. Geophys. Res., 103, 8651-8666, 1998.

Niwano, M., Yamazaki, K., and Shiotani, M.: Seasonal and QBO variations of ascent rate in the tropical lower stratosphere as inferred from UARS HALOE trace gas data, J. Geophys. Res., 108, 4794, doi:10.1029/2003JD003871, 2003.

Schoeberl, M. R., Douglass, A. R., Zhu, Z., and Pawson, S.: A comparison of the lower stratospheric age spectra derived from a global circulation model and two data assimilation systems, J. Geophys. Res., 108, doi:10.1029/2002JD002652, 2003.

Swinbank, R. and O'Neill, A.: A stratosphere-troposphere data assimilation system, Mon. Weather Rev., 122, 686-702, 1994.

Uppala, S. M., Kållberg, P., Simmons, A. J., et al.: The ERA40 reanalysis, Quart. J. Roy. Meteorol. Soc., 131, 2961-3012, doi:10.1256/qj.04.176, 2005.

van Noije, T. P. C., Eskes, H. J., van Weele, M., and van Velthoven, P.: Implications of the enhanced Brewer-Dobson circulation in ERA-40 for the stratosphere-troposphere exchange of ozone in global chemistry-transport models, J. Geophys. Res., 109, D19308, doi:101029/2004JD004586, 2004.

Weaver, C. J., Douglass, A. R., and Rood, R. B.: Lamination frequencies as a diagnostic for horizontal mixing in a $3 \mathrm{D}$ transport model, J. Atmos. Sci., 57, 247-261, 2000. 